Bio-grafía. Escritos sobre la Biología y su Enseñanza. ISSN 2027-1034

Número Extraordinario. p.p. 65-75

Memorias del Primer encuentro ambiental Universidad, ambiente y sustentabilidad: experiencias y prácticas.

\title{
EL SEMILLERO-CLUB DE INVESTIGACIÓN SOBRE EDUCACIÓN EN QUÍMICA VERDE Y SUSTENTABILIDAD AMBIENTAL - EDUQVERSA. UNA PROPUESTA EN CONSTRUCCIÓN
}

\author{
Rozo Nicolás ${ }^{1}$ \\ Guevara Edward ${ }^{1}$ \\ Franco Ricardo ${ }^{2}$
}

\section{Resumen}

Habida cuenta del compromiso institucional de la Universidad Pedagógica Nacional con la Sustentabilidad Ambiental, que se explicita en el eje No. 4 del Plan de Desarrollo Institucional "Una universidad comprometida con la formación de maestros para una Colombia en paz", el Grupo de Investigación interinstitucional Representaciones y Conceptos Científicos IREC ha venido dinamizando la conformación y el desarrollo del Semillero-Club de investigación: Educación en Química Verde para la Sustentabilidad Ambiental - EduQVersa, integrado por profesores en formación inicial, continua y en ejercicio de la Facultad de Ciencia y Tecnología de la UPN.

Así, la presente propuesta de investigación enfoca su atención especialmente en el fomento de procesos de investigación formativa conducentes al fortalecimiento de las dimensiones investigativa y ambiental en la formación inicial de profesores de ciencias de la Universidad Pedagógica Nacional, a partir del desarrollo de un semillero-club de

${ }^{1}$ Universidad Pedagógica Nacional. - Profesores en formación inicial - Departamento de química - Semillero-club de Investigación EduQversa - Grupo de investigación IREC edqversa@gmail.com e Integrantes del semillero EduQversa.

${ }^{2}$ Universidad Pedagógica Nacional. - Docente en ejercicio - Departamento de química Semillero de Investigación EduQversa - Grupo de investigación IREC = rfranco@pedagogica.edu.co 
Bio-grafía. Escritos sobre la Biología y su Enseñanza. ISSN 2027-1034

Número Extraordinario. p.p. 65-75

Memorias del Primer encuentro ambiental Universidad, ambiente y sustentabilidad: experiencias y prácticas.

investigación en cuyo marco se propone el desarrollo de iniciativas de investigación e innovación individuales y colectivas acerca de química verde, energías alternativas y sustentabilidad ambiental. En este contexto, se busca la consolidación de un espacio extracurricular que permita articular la dinámica del semillero-club y los procesos formativos propios del currículo, en el marco de la reflexión, la crítica y la acción frente a la temática central, a partir del reconocimiento de situaciones y problemáticas socioambientales relevantes para la formación inicial de profesores de ciencias, y abordarlas en el contexto de la sustentabilidad ambiental.

\section{Palabras clave}

Química verde, Sustentabilidad ambiental, formación investigativa, Semilleroclub de investigación.

\section{Abstract}

Given the institutional commitment of the National Pedagogical University with the Environmental Sustainability, which is made explicit in the axis No. 4 Institutional Development Plan "A university committed to training teachers for a Colombia at peace," the Interagency Research representations and Scientific Concepts IREC has been streamlining the creation and development of Semillero-Club research: Education on Green Chemistry for Environmental Sustainability - EduQVersa, composed of teachers in initial, ongoing training and exercise of the Faculty of Science and Technology UPN.

Thus, the present research proposal focuses its attention on the promotion of processes conducive to strengthening research and environmental dimensions in the initial training of science teachers of the National Pedagogical University formative research, from the development of a semillero- research club under which the development of research initiatives 
Bio-grafía. Escritos sobre la Biología y su Enseñanza. ISSN 2027-1034

Número Extraordinario. p.p. 65-75

Memorias del Primer encuentro ambiental Universidad, ambiente y sustentabilidad: experiencias y prácticas.

and individual and collective innovation on green chemistry, alternative energy and environmental sustainability is proposed. In this context, the consolidation of an extracurricular space to articulate the dynamics of seedclub and proper training processes of the curriculum, as part of the reflection, critique and action against the central theme, from seeks recognition of situations and relevant social and environmental issues for the initial training of science teachers, and addressed in the context of environmental sustainability.

\section{Keywords}

Green Chemistry, Environmental Sustainability, research training, research Semillero-club.

\section{OBJETIVOS}

Fortalecer la dimensión ambiental en la formación inicial de profesores de ciencias de la Universidad Pedagógica Nacional, a partir del desarrollo de un semillero-club de investigación en educación sobre química verde y energías alternativas para la sustentabilidad ambiental.

\section{OBJETIVOS ESPECÍFICOS:}

- Desarrollar iniciativas de investigación e innovación individuales y colectivas acerca de química verde, energías alternativas y sustentabilidad ambiental.

- Consolidar un espacio extracurricular que permita articular la dinámica del semillero-club y los procesos formativos propios del currículo, en el marco de la reflexión, la crítica y la acción frente a la sustentabilidad ambiental. 
Bio-grafía. Escritos sobre la Biología y su Enseñanza. ISSN 2027-1034

Número Extraordinario. p.p. 65-75

Memorias del Primer encuentro ambiental Universidad, ambiente y sustentabilidad: experiencias y prácticas.

- Reconocer situaciones y problemáticas socioambientales relevantes para la formación inicial de profesores de ciencias, y abordarlas en el contexto de la sustentabilidad ambiental.

\section{MARCO TEÓRICO}

Partiendo del reconocimiento de la necesidad de adelantar procesos de formación investigativa en la Facultad de Ciencia y Tecnología de la Universidad Pedagógica Nacional, durante el periodo comprendido entre 2011 II y 2015 I, se desarrolló el proyecto de facultad: "Formación de jóvenes investigadores en la Facultad de Ciencia y Tecnología de la UPN" - FCT1911 (Franco, Martínez, Pareja, Guerrero, Vargas y López, 2015), entre cuyos resultados centrales se destacan la conformación de semilleros de investigación por departamentos, el desarrollo de iniciativas estudiantiles de investigación formativa y la socialización de las mismas en eventos locales, regionales y nacionales.

En particular, a partir del proyecto en mención se conformó y se consolidó el Semillero de Investigación en Educación, Pedagogía y Didáctica de la Química - DIDAGOKHEMIA, el cual fue reconocido institucionalmente por la UPN mediante el proyecto de investigación DQU-001-S-15, desarrollado durante los periodos 2015 II y 2016 I, fomentando así la formación investigativa de profesores en el Departamento de Química de la UPN y propiciando algunas iniciativas de investigación formativa en múltiples campos del conocimiento en educación en ciencias, entre ellas algunas relacionadas con el enfoque de química verde y energías alternativas (Ortiz, Ordóñez, Suárez, Gallo, Franco y Martínez, 2016).

En lo tocante a la formación investigativa de los futuros profesores de ciencias en general y de química en particular, a partir del proyecto de 
Bio-grafía. Escritos sobre la Biología y su Enseñanza. ISSN 2027-1034

Número Extraordinario. p.p. 65-75

Memorias del Primer encuentro ambiental Universidad, ambiente y sustentabilidad: experiencias y prácticas.

investigación: DQU-358-13 "La dimensión investigativa en la formación inicial de profesores de química. El caso de la UPN", Franco, Gallego y Pérez (2015) reconocen múltiples problemáticas, poniendo de manifiesto la necesidad de incentivar la investigación como un proceso formativo y constructivo que ha de agenciar la consolidación de una comunidad académica que produzca conocimientos educativos y culturales de manera permanente (Franco, Ramírez y González, 2009). Desde esta perspectiva, se hace necesario el desarrollo y la consolidación de espacios formativos como los denominados de semilleros de investigación (Franco Ávila y Franco Moreno, 2009).

En el contexto de la dimensión ambiental en la formación inicial de profesores de ciencias, el análisis de procesos de educación ambiental en comunidades vulnerables, pone de presente la necesidad de fomentar espacios que permitan vincular dicha dimensión en la preparación de los futuros maestros (Pérez y Franco, 2013). En esta dirección, Rojas y Usaquén (2014) desarrollaron una investigación tendiente a fomentar el reverdecimiento escolar en la Institución Educativa Distrital Tomás Carrasquilla de Bogotá, coincidiendo con la urgencia de una formación de profesores de química para la sustentabilidad ambiental.

Los anteriores aportes, junto con el devenir de la línea de investigación Educación en ciencias para la sustentabilidad ambiental y la apropiación social del conocimiento científico, del Grupo IREC, hicieron posible problematizar el abordaje de la sustentabilidad ambiental en la formación inicial de profesores de química, vinculando así algunos tópicos del enfoque emergente de química verde (Reyes-Sánchez, 2012) en espacios académicos del programa de Licenciatura en Química, tales como Teorías 
Bio-grafía. Escritos sobre la Biología y su Enseñanza. ISSN 2027-1034

Número Extraordinario. p.p. $65-75$

Memorias del Primer encuentro ambiental Universidad, ambiente y sustentabilidad: experiencias y prácticas.

Químicas II y Educación Ambiental, en los que se realizaron microproyectos por parte de los estudiantes.

Así, en el periodo 2015-I se formuló el curso electivo de facultad: "Química verde y energías alternativas para los profesores de ciencias", generando algunas producciones que fueron socializadas en el marco del II Workshop de investigación en la formación inicial de profesores de ciencias y II Encuentro de PGI en la interfaz Universidad-Escuela realizado en Bogotá en octubre de 2015, y reconociendo así la necesidad de investigar y profundizar en este campo de la ciencia, como una posibilidad para fomentar la sustentabilidad ambiental en la formación inicial de profesores de ciencias (Mascarell y Vilches, 2016).

La comprensión sobre química verde y energías alternativas para los profesores de ciencias que se apropia en el semillero EduQVersa, se encuentra fundamentada por los doce principios de química verde, sus representaciones y concepciones, al igual que sus lineamientos en el momento de aplicar algún proceso de producción mediante TPL o producción textual en artículos científicos.

\section{RESULTADOS}

Lo anterior se recoge, en buena medida, en la conformación del Semilleroclub de investigación EduQVersa, cuya dinámica hoy da cuenta de los siguientes productos y actividades desarrolladas:

\begin{tabular}{|l|l|}
\hline Actividades / productos & Medio de divulgación \\
\hline $\begin{array}{l}\text { Desarrollo de microproyectos } \\
\text { sobre aplicaciones de química }\end{array}$ & $\begin{array}{l}\text { Cursos de Teorías Químicas II y } \\
\text { Educación Ambiental (Programa de }\end{array}$ \\
\hline
\end{tabular}


Bio-grafía. Escritos sobre la Biología y su Enseñanza. ISSN 2027-1034

Número Extraordinario. p.p. 65-75

Memorias del Primer encuentro ambiental Universidad, ambiente y sustentabilidad: experiencias y prácticas.

\begin{tabular}{|l|l|}
\hline verde. & Licenciatura en Química). \\
\hline Proyectos de investigación de & Trabajo de grado. \\
licenciatura sobre el enfoque. & \\
(Florián Ardila, 2015) & \\
\hline Caractización & \\
\hline
\end{tabular}

\begin{tabular}{|lr|lr|}
\hline Caracterización abordaje del & Congresorar Internacional de \\
enfoque de química verde en las & enseñanza de las ciencias- CECAR \\
revistas especializadas en & 2016. Sincelejo, Colombia.
\end{tabular}

didáctica de las ciencias. Estudio cienciométrico.

(Franco, Ordóñez y Rozo, 2016).

Producción de un combustible alternativo, a partir de cáscaras de naranja minimizando la emisión de gases de invernadero Angie Carolina Avendaño Quintero, María Isabel Sánchez Ocampo, Loren Dahana Limas Castellanos, Manuel Alejandro Ordoñez Castillo La fitoremediación para reducir el II Workshop de investigación en la impacto ambiental que genera el formación inicial de profesores de mercurio en los ecosistemas ciencias y II Encuentro de PGI en la acuáticos: un microproyecto de interfaz Universidad-Escuela. Bogotá, química verde. 2015

María Alejandra Velasco, María José Cortés, Jhon Alexander Gallo, Alejandra Perdomo La química verde: ¿un campo de Viernes de la Didáctica de las frontera en la didáctica de la ciencias 
Bio-grafía. Escritos sobre la Biología y su Enseñanza. ISSN 2027-1034

Número Extraordinario. p.p. $65-75$

Memorias del Primer encuentro ambiental Universidad, ambiente y sustentabilidad: experiencias y prácticas.

\begin{tabular}{|c|c|}
\hline $\begin{array}{l}\text { química? } \\
\text { Ricardo Andrés Franco }\end{array}$ & Bogotá, junio de 2015. \\
\hline $\begin{array}{l}\text { Producción de Biogás a partir de } \\
\text { un biodigestor. Una iniciativa de } \\
\text { química verde. } \\
\text { Nicolás Rozo, Adrián Ortiz, } \\
\text { Fernanda Arango. }\end{array}$ & $\begin{array}{l}\text { Viernes de la Didáctica de las } \\
\text { ciencias } \\
\text { Bogotá, junio de } 2015 .\end{array}$ \\
\hline $\begin{array}{l}\text { Producción de Biodiesel mediante } \\
\text { aceite reutilizado. Una aplicación } \\
\text { de la química verde. Edward } \\
\text { Guevara, Catalina Cruz, Laura } \\
\text { López y Katherine Sánchez. }\end{array}$ & $\begin{array}{l}\text { Viernes de la Didáctica de las } \\
\text { ciencias } \\
\text { Bogotá, junio de } 2015 .\end{array}$ \\
\hline $\begin{array}{l}\text { Práctica experimental, con un } \\
\text { enfoque de química verde, para la } \\
\text { elaboración de crema dental. } \\
\text { Julieth Reina Hernández y María } \\
\text { Isabel Sánchez }\end{array}$ & $\begin{array}{l}\text { Viernes de la Didáctica de las } \\
\text { ciencias } \\
\text { Bogotá, junio de } 2015 \text {. }\end{array}$ \\
\hline $\begin{array}{l}\text { Incorporación del enfoque de } \\
\text { química verde en la educación } \\
\text { secundaria: Una estrategia } \\
\text { curricular. } \\
\text { Paola Andrea López Moreno. }\end{array}$ & $\begin{array}{l}\text { Proyecto de PPDQ } \\
\text { Proyecto de grado Lic. Química (en } \\
\text { desarrollo) }\end{array}$ \\
\hline
\end{tabular}


Bio-grafía. Escritos sobre la Biología y su Enseñanza. ISSN 2027-1034

Número Extraordinario. p.p. 65-75

Memorias del Primer encuentro ambiental Universidad, ambiente y sustentabilidad: experiencias y prácticas.

\section{METODOLOGÍA}

El Semillero-club de investigación EduQVersa se compromete desarrolla las siguientes acciones en su dinámica de trabajo colectivo:

- -Encuentros semanales (periódicos) entre profesores en formación inicial y en ejercicio para la discusión, la construcción de aproximaciones conceptuales y metodológicas y el diálogo de saberes.

- Articulación de las actividades y desarrollos del semillero con espacios académicos de los programas de formación de la Facultad de Ciencia y Tecnología, mediante la participación activa de estudiantes y profesores de los mismos.

- Organización y desarrollo de encuentros de Semilleros de Investigación en el área.

- Organización y desarrollo de sesiones de los Viernes de la Didáctica de las Ciencias dedicadas exclusivamente a la temática del proyecto.

- Conformación de una red de semilleros de investigación sobre educación en ciencias aplicadas a la sustentabilidad ambiental. 
Bio-grafía. Escritos sobre la Biología y su Enseñanza. ISSN 2027-1034

Número Extraordinario. p.p. 65-75

Memorias del Primer encuentro ambiental Universidad, ambiente y sustentabilidad: experiencias y prácticas.

\section{CONCLUSIONES}

Dicho semillero-club, como espacio extracurricular en el que se propicia la formación investigativa, se hace necesario para fortalecer las dimensiones ambiental e investigativa en la formación inicial de profesores de ciencias, desde la perspectiva de la educación en química verde para la sustentabilidad ambiental, permitiendo al futuro profesor acercarse a las problemáticas socioambientales contemporáneas, fomentando la reflexión, la crítica y la acción frente a las mismas, desde su quehacer como maestro en los diferentes niveles del sistema educativo. Así, las siguientes preguntas orientan el horizonte de sentido del Semillero-club EduQVersa:

¿Cuáles son los aportes que se generan en la dimensión ambiental de la formación inicial de profesores de ciencias de la Universidad Pedagógica Nacional al desarrollar un semillero-club de investigación en química verde y energías alternativas para la sustentabilidad?

¿Desde qué perspectivas conceptuales y metodológicas es posible fomentar una educación en química para la sustentabilidad ambiental, vinculando el enfoque de química verde y energías alternativas a través de un semilleroclub de investigación?

\section{REFERENCIAS BIBLIOGRÁFICAS}

Franco, R.A, Ramírez, J y González, K.V. (2009). Semilleros de investigación vs comunidades científicas: ¿moda o necesidad? TECKNE. 6 (2), 35-37.

Franco Ávila, M., \& Franco Moreno, R. (2009). El semillero de investigadores de la UPN desde la perspectiva de organización en red: Un espacio para la interlocución investigativa. En J. Blanco, \& J. Piedrahita, III encuentro regional de universidades públicas. Escenarios para la construcción de comunidades académicas y científicas. (págs. 23-32). Bogotá: Universidad Colegio Mayor de Cundinamarca. 
Bio-grafía. Escritos sobre la Biología y su Enseñanza. ISSN 2027-1034

Número Extraordinario. p.p. $65-75$

Memorias del Primer encuentro ambiental Universidad, ambiente y sustentabilidad: experiencias y prácticas.

Franco, R., Martínez, L., Pareja, A., Guerrero, N., Vargas, C y López, C. (2015). Proyecto de facultad: FCT-1911. Formación de jóvenes investigadores en la Facultad de Ciencia y Tecnología de la UPN. Informe de vigencia $2015 \mathrm{I}$.

Mascarell, I y Vilches, A (2016). Química Verde y Sostenibilidad en la educación en ciencias en secundaria. Enseñanza de las ciencias. 34 (2), 2542.

Ortiz, C., Ordóñez, L., Suárez, J., Gallo, J., Franco, R.A y Martínez, L. (2016). El semillero de investigación DIDAGOKHEMIA: caracterización de los aportes en la formación investigativa de profesores de química. V Congreso Internacional de Investigación en Educación en Ciencias Naturales y Tecnología - EDUCYT. Neiva, 24 al 26 de agosto de 2016.

Pérez, O y Franco, R (2013). Análisis del proceso comunitario: "la gente del Tunjuelo" como un escenario de formación ambiental, en el pantano la libélula. Biografía. Escritos sobre la biología y su enseñanza. No. Extra: Memorias del VII Encuentro Nacional de Experiencias en la Enseñanza de la Biología y la Educación Ambiental y II Congreso Nacional de Investigación en la Enseñanza de la Biología. 3-10.

Reyes-Sánchez. (2012). Aporte de la química verde a la construcción de una ciencia socialmente responsable. Educación Química. 23(2), 222-229. 